\title{
Molecular detection and genotypic characterization of Toxoplasma gondii infection in bats in four provinces of China
}

Si-Yuan Qin ${ }^{1,3}$, Wei Cong ${ }^{1,3}$, Ye Liu'², Nan Li ${ }^{2}$, Ze-Dong Wang ${ }^{2}$, Fu-Kai Zhang ${ }^{1}$, Si-Yang Huang ${ }^{1}$, Xing-Quan Zhu ${ }^{1,3,4^{*}}$ and Quan Li $2^{2^{*}}$

\begin{abstract}
Background: Toxoplasma gondii is an intracellular protozoan parasite that infects a wide variety of warm-blooded hosts, including humans. Limited information about T. gondii infection in bats is available in China. The objective of the present study was to determine prevalence and genetic characterization of T. gondii infection in bats in Jilin, Liaoning, Jiangxi and Guangdong provinces, China.

Methods: During May 2005 to August 2013, bats were sampled from Jilin, Liaoning, Jiangxi, and Guangdong provinces, China, and liver tissues were collected for the detection of T. gondii by a nested PCR targeting the B1 gene. The positive samples were genotyped at 11 genetic markers (SAG1, 5'-and 3'-SAG2, alternative SAG2, SAG3, BTUB, GRA6, L358, PK1, C22-8, C29-2, and Apico) using multilocus polymerase chain reaction-restriction fragment length polymorphism (PCR-RFLP).

Results: A total of 626 bats representing 10 species were examined for T. gondii infection, 38 (6.1\%) were tested positive with by PCR, 8 positive DNA samples were completely genotyped, of which 3 samples (2 from Cynopterus sphinx, and 1 from Murina leucogaster) represented ToxoDB\#10, and 5 samples ( 2 from Murina leucogaster, 2 from Myotis chinensis, and 1 from Rhinolophus ferrumequinum) belonged to ToxoDB\#9 (http://toxodb.org/toxo/).

Conclusions: The present study revealed an overall T. gondii prevalence of $6.1 \%$ in bats from Jilin, Liaoning, Jiangxi and Guangdong provinces in China, and reported two T. gondii genotypes (ToxoDB\#9 and \#10) having a wide geographical distribution in China. These results provide new genetic information about $T$. gondii infection in bats, and have implications for better understanding of the genetic diversity of T. gondii in China and elsewhere.
\end{abstract}

Keywords: Toxoplasma gondii, Bats, Prevalence, Genetic characterization, China

\section{Background}

Toxoplasma gondii is an obligate intracellular protozoan parasite with a worldwide distribution, which can infect a wide variety of warm blooded animals and humans. Approximately one third of the world population and $7.9 \%$ of population in China are seropositive for $T$.

\footnotetext{
* Correspondence: xingquanzhu1@hotmail.com; liuquan1973@hotmail.com ${ }^{1}$ State Key Laboratory of Veterinary Etiological Biology, Key Laboratory of Veterinary Parasitology of Gansu Province, Lanzhou Veterinary Research Institute, Chinese Academy of Agricultural Sciences, Lanzhou, Gansu Province 730046, PR China

${ }^{2}$ Military Veterinary Institute, Academy of Military Medical Sciences, Key Laboratory of Jilin Province for Zoonosis Prevention and Control, Changchun, Jilin Province 130122, PR China

Full list of author information is available at the end of the article
}

gondii antibodies [1-3]. T. gondii infection can cause serious diseases in the developing fetus and immunocompromised individuals [4]. It is transmitted to humans and animals via ingesting $T$. gondii tissue cysts from undercooked meat, by consuming water or food contaminated with $T$. gondii oocysts, or through transplacental transmission $[5,6]$.

Bats are considered as important natural reservoir of many zoonotic viruses, such as rabies virus, Hantavirus, Marburg virus, Nipah virus, Ebola virus and severe acute respiratory syndrome coronaviruses $[7,8]$. Besides viruses, bacteria, fungi, and protozoa have been detected in bats, and can potentially be transmitted to humans [9-11]. Bats are important to public health, as they live 
in different habitats and have high mobility and the possible interactions with humans.

Although bats play significant roles in the transmission of some important zoonotic diseases, there were only limited reports on T. gondii infection in bats. The prevalence of $T$. gondii infection was $10.4 \%$ in British bats [12], and 29.3\% in bats in Myanmar [13]. Additionally, there were two cases of toxoplasmosis described in captive bats in Australia [14], and several T. gondii strains have been isolated in Kazakhstan and in Brazil $[15,16]$. In China, $T$. gondii seroprevalence in five bat species in four provinces was detected using modified agglutination test (MAT) [17]. However, there was only one previous report on genotypes of $T$. gondii in bats in Yunnan and Guangxi, southern China [18]. In the present study, we determined the prevalence and characterized $T$. gondii isolates in bats in other four provinces of China.

\section{Methods}

\section{Ethics statement}

This study was approved by the Ethics Committee of Military Veterinary Institute, Academy of Military Medical Sciences. Bats were handled in accordance with good animal practices required by the Animal Ethics Procedures and Guidelines of the People's Republic of China.

\section{Study sites and bat collection}

The study was conducted in four provinces of China, including Jilin $\left(41^{\circ}-46^{\circ} \mathrm{N}, 122^{\circ}-131^{\circ} \mathrm{E}\right)$, Liaoning $\left(38^{\circ}-43^{\circ}\right.$ $\left.\mathrm{N}, 118^{\circ}-125^{\circ} \mathrm{E}\right)$, Jiangxi $\left(24^{\circ}-30^{\circ} \mathrm{N}, 113^{\circ}-118^{\circ} \mathrm{E}\right)$, and Guangdong $\left(20^{\circ}-25^{\circ} \mathrm{N}, 109^{\circ}-117^{\circ} \mathrm{E}\right)$ from May 2005 to August 2013. Jilin and Liaoning provinces are located in northeastern of China, where the average temperature is lower than other two provinces. Jiangxi province, located in southeast China, has an average annual temperature of $11.6-19.6^{\circ} \mathrm{C}$. Guangdong province lies on southern edge of mainland, with an average annual temperature of $22.8^{\circ} \mathrm{C}$. Bats were captured at roosts with hand nets, and identified to species in the field.

\section{DNA extraction and PCR detection}

Bats were euthanized, and liver tissues were collected for genomic DNA extraction using the TIANamp Genomic DNA kit (TianGen, Beijing, China). T. gondii infection was examined by a semi-nested PCR targeting the B1 gene as described elsewhere [19-21]. The positive samples were used further for genetic characterization.

\section{Genetic characterization of $T$. gondii}

Multilocus PCR-RFLP was conducted to genetically characterize $T$. gondii in bats, using 11 genetic markers (i.e., SAG1, 5' -and 3'-SAG2, alternative SAG2, SAG3, BTUB, GRA6, c22-8, c29-2, L358, PK1, and Apico) as described previously $[12,18]$. Briefly, the target sequences were first amplified by multiplex PCR using external primers for all 11 markers. Nine reference strains, namely GT1, PTG, CTG, MAS, TgCgCa1, TgCatBr5, TgCatBr64, TgRsCr1, and TgWtdSc40, were used as the positive controls (Table 1). The PCR amplification was performed using a thermal cycler (PTC 200, Bio-RAD). The T. gondii B1-positive DNA sample was incubated at $95^{\circ} \mathrm{C}$ for 5 min to activate the DNA polymerase, then 30 cycles of PCR at $95^{\circ} \mathrm{C}$ for $30 \mathrm{~s}, 55^{\circ} \mathrm{C}$ for $60 \mathrm{~s}$ and $72^{\circ} \mathrm{C}$ for $90 \mathrm{~s}$, and then at $72^{\circ} \mathrm{C}$ for $7 \mathrm{~min}$. Then $1 \mu \mathrm{l}$ of the products were used as template DNA for nested PCR with internal primers for each marker, respectively. The nested PCR products were digested with restriction enzymes for $2 \mathrm{~h}$, and the restriction fragments were resolved in $2.5 \%$ agarose gel to distinguish single nucleotide polymorphisms (SNPs) using a gel document system (UVP GelDoc-It ${ }^{\mathrm{tw}}$ Imaging System, Cambridge, UK).

\section{Results and discussion}

A total of 626 bats, belonging to ten species of seven genera, were collected in the present study (Table 2). The dominant bat species was Murina leucogaster in Jilin, Myotis ricketti in Liaoning, M. leucogaster and Myotis chinensis in Jiangxi, and Hipposideros larvatus and Cynopterus sphinx in Guangdong.

Of 626 examined bat samples, 38 samples were tested positive for the $T$. gondii $\mathrm{B} 1$ gene by PCR amplification, including 8 of 222 (3.6\%) M. leucogaster, 10 of 139 (7.2\%) M. chinensis, 2 of 56 (3.6\%) M. ricketti, 6 of 43 (14.0\%) Rhinolophus ferrumequinum, 1 of 54 (1.9\%) Cynopterus sphinx, 3 of 30 (10.0\%) Rousettus leschenaultia, 8 of 67 (11.9\%) H. larvatus (Table 2). Only three species, including Plecotus auritus in Jilin, M. chinensis in Liaoning, and Hipposideros armiger in Guangdong, were detected negative, probably due to the small number of samples, or the low prevalence.

The results of the present study demonstrated that $T$. gondii infection in bats was widely distributed in Jilin, Liaoning, Jiangxi and Guangdong provinces, with prevalence ranging from $3.7 \%$ to $7.3 \%$. Other surveys have shown an overall prevalence of $6.7 \%$ in bats in Yunnan, and $20.3 \%$ in Guangxi by a nested PCR [18], and a seroprevalence of $18.4 \%$ in five bat species in Anhui, Hubei, Guangdong, and Guangxi by MAT [17]. In other countries, the prevalence of $T$. gondii infection was found in 10.4\% British bats using a SAG1-PCR [12], and in 29.3\% bats in Myanmar by a nested B1-PCR [13]. The difference may be related to bats species, study regions, and the detection methods.

The present study revealed that $T$. gondii prevalence was higher in bats in southern China (Guangdong, and Jiangxi) than in northern China (Jilin, and Liaoning). The possible reason is that warm and humid environment in 
Table 1 Genetic characterization of Toxoplasma gondii isolates from bats in Jilin, Jiangxi and Guangdong provinces, China

\begin{tabular}{|c|c|c|c|c|c|c|c|c|c|c|c|c|c|c|c|}
\hline Isolate ID & Host & Tissue & Location & SAG1 & $5^{\prime}+3^{\prime}$ SAG2 & Alternative SAG2 & SAG3 & BTUB & GRA6 & c22-8 & c29-2 & L358 & PK1 & Apico & Genotype \\
\hline GT1 & Goat & & United States & I & I & 1 & I & I & I & I & I & I & I & I & Reference, Type I, ToxoDB \#10 \\
\hline PTG & Sheep & & United States & $\|/\| \|$ & $\|$ & $\|$ & $\|$ & $\|$ & $\|$ & $\|$ & $\|$ & $\|$ & $\|$ & $\|$ & Reference, Type II, ToxoDB \#1 \\
\hline CTG & Cat & & United States & $\|/\|$ & III & III & III & III & III & III & III & III & III & III & Reference, Type III, ToxoDB \#2 \\
\hline MAS & Human & & France & $\mathrm{u}-1^{*}$ & । & $\|$ & III & III & III & $\mathrm{u}-1^{*}$ & I & । & III & । & Reference, ToxoDB \#17 \\
\hline TgCgCa1 & Cougar & & Canada & I & $\|$ & $\|$ & III & $\|$ & $\|$ & $\|$ & $\mathrm{u}-1^{*}$ & I & $\mathrm{u}-2^{*}$ & । & Reference, ToxoDB \#66 \\
\hline TgCatBr5 & Cat & & Brazil & । & III & III & III & III & III & । & । & । & $\mathrm{u}-1^{*}$ & । & Reference, ToxoDB \#19 \\
\hline TgWtdSc40 & WTD & & USA & $u-1$ & $\|$ & $\|$ & $\|$ & $\|$ & $\|$ & $\|$ & $\|$ & । & $\|$ & I & Type 12, ToxoDB \#5 \\
\hline TgCatBr64 & Cat & & Brazil & । & । & $u-1$ & III & III & III & $u-1$ & । & III & III & । & Reference, ToxoDB \#111 \\
\hline TgRsCr1 & Toucan & & Costa Rica & $\mathrm{u}-1$ & । & ॥ & III & । & III & $u-2$ & । & I & III & 1 & Reference, ToxoDB \#52 \\
\hline TgBatJL1- TgBatJL2 & $M C$ & Liver & Jilin, China & $u-1$ & $\|$ & $\|$ & III & III & $\|$ & $\|$ & III & $\|$ & $\|$ & । & ToxoDB \#9 \\
\hline TgBatJL3 & $M L$ & Liver & Jilin, China & । & । & । & । & । & I & I & I & । & । & । & ToxoDB \#10 \\
\hline TgBatJL4 & $M L$ & Liver & Jilin, China & । & I & । & । & nd & I & I & । & । & nd & nd & $=$ nd \\
\hline TgBatJX5- TgBatJX7 & RF & Liver & Jiangxi, China & $u-1$ & $\|$ & $\|$ & III & III & $\|$ & $\|$ & III & $\|$ & $\|$ & I & ToxoDB \#9 \\
\hline TgBatGD8 & CS & Liver & Guangdong, China & I & I & । & I & I & I & 1 & nd & I & I & nd & nd \\
\hline TgBatGD9, TgBatGD10 & CS & Liver & Guangdong, China & I & । & I & । & । & । & I & । & । & । & । & ToxoDB \#10 \\
\hline TgBatGD11 & CS & Liver & Guangdong, China & nd & 1 & nd & 1 & I & I & 1 & 1 & 1 & nd & nd & nd \\
\hline
\end{tabular}

${ }^{*} \mathrm{u}-1$ and $\mathrm{u}-2$ represent unique RFLP genotypes, respectively.

nd: not determined.

WTD: White-tailed Deer; CS: Cynopterus sphinx; ML: Murina leucogaster; MC: Myotis chinensis; RF: Rhinolophus ferrumequinum. 
Table 2 Prevalence of Toxoplasma gondii infection in bats in four provinces, China

\begin{tabular}{|c|c|c|c|c|}
\hline Province & Bat species & No. of examined $(\%)^{*}$ & No. of positive & Prevalence (\%) \\
\hline \multirow[t]{5}{*}{ Jilin } & Murina leucogaster & $140(22.4)$ & 6 & 4.3 \\
\hline & Myotis chinensis & $27(4.3)$ & 3 & 11.1 \\
\hline & Plecotus auritus & $2(0.3)$ & 0 & 0.0 \\
\hline & Rhinolophus ferrumequinum & $8(1.3)$ & 1 & 12.5 \\
\hline & Subtotal & $177(28.3)$ & 10 & 5.6 \\
\hline \multirow[t]{4}{*}{ Liaoning } & Myotis chinensis & $9(1.4)$ & 0 & 0.0 \\
\hline & Myotis ricketti & $56(8.9)$ & 2 & 3.6 \\
\hline & Rhinolophus ferrumequinum & $17(2.7)$ & 1 & 5.9 \\
\hline & Subtotal & $82(13.1)$ & 3 & 3.7 \\
\hline \multirow[t]{4}{*}{ Jiangxi } & Murina leucogaster & $82(13.1)$ & 2 & 2.4 \\
\hline & Myotis chinensis & $103(16.5)$ & 7 & 6.8 \\
\hline & Rhinolophus ferrumequinum & $18(2.9)$ & 4 & 22.2 \\
\hline & Subtotal & $203(32.4)$ & 13 & 6.4 \\
\hline \multirow[t]{6}{*}{ Guangdong } & Cynopterus sphinx & $54(8.6)$ & 1 & 1.9 \\
\hline & Hipposideros armiger & $4(0.6)$ & 0 & 0.0 \\
\hline & Hipposideros larvatus & $67(10.7)$ & 8 & 11.9 \\
\hline & Hipposideros pomona & $9(1.4)$ & 0 & 0.0 \\
\hline & Rousettus leschenaulti & $30(4.8)$ & 3 & 10.0 \\
\hline & Subtotal & $164(26.2)$ & 12 & 7.3 \\
\hline Total & & 626 & 38 & 6.1 \\
\hline
\end{tabular}

*The percent accounts for the total bats.

southern China is more suitable for survival of $T$. gondii oocysts [1].

The $38 T$. gondii-positive bat samples were directly genotyped, and only 8 positive DNA samples were completely genotyped, possibly due to low DNA concentration. Of which, 3 samples ( 2 from $C$. sphinx, and 1 from M. leucogaster) represented ToxoDB Genotype $\# 10$, and 5 samples (2 from M. leucogaster, 2 from $M$. chinensis, and 1 from $R$. ferrumequinum) belonged to ToxoDB Genotype \#9 (Table 1).

Two genotypes, namely ToxoDB\#10 and ToxoDB\#9, were found in bats in this study, which was consistent with a previous study [18], showing a limited diversity of T. gondii genotypes in bats in China. Unfortunately, T. gondii genotype in bats was not successfully identified in Liaoning, probably due to the small size of sampled bats and low intensity of infection.

Recent studies have demonstrated that bats may share the same $T$. gondii genotypes as in wild and domestic animals, and humans. Although several genotypes of $T$. gondii have been described in China, there are two main genotypes, including types I (ToxoDB\#10) and an atypical genotype ToxoDB\#9. In particular, genotype \#9 has been reported in Microtus fortis in Jilin province [22], pigs in Jiangxi and Yunnan provinces [20,23], cats in
Beijing, Yunnan and Guangdong provinces [24-26], bats in Yunnan and Guangxi provinces [18], and humans $[27,28]$, suggesting that the genotype ToxoDB\#9 is the most common lineage in mainland China. It is not only present in China, but also in Vietnam and Sri Lanka, which indicated a widespread distribution in Eastern Asia [29]. ToxoDB\#10 is also common in China, found in Plateau pikas and Qinghai voles in Qinghai [19], $M$. fortis in Jilin [22], tree sparrows in Fujian [30], pigs in Henan, Hubei, Hunan, and Jiangsu [27,31], sheep in Qinghai [27], and human in Shanghai [27]. These results have shown a wide distribution of the two genotypes identified from bats in China.

\section{Conclusions}

The present study revealed an overall $T$. gondii prevalence of $6.1 \%$ in bats from Jilin, Liaoning, Jiangxi and Guangdong provinces, China, and reported two $T$. gondii genotypes (ToxoDB\#9 and \#10). The wide geographical distribution of the two genotypes implied an important role of bats in transmission of $T$. gondii. These results provide new genetic information about T. gondii infection in bats, and have implications for better understanding of the genetic diversity of $T$. gondii in China. 


\section{Competing interests}

The authors declare that they have no competing interests.

\section{Authors' contributions}

$\mathrm{XQZ}$ and $\mathrm{QL}$ conceived and designed the study, and critically revised the manuscript. SYQ, WC, YL, NL, ZDW and FKZ collected the sample, performed the experiments, analyzed the data and drafted the manuscript. SYH helped in study design, study implementation and manuscript revision. All authors read and approved the final manuscript.

\section{Acknowledgments}

Project support was provided in part by the National Natural Science Foundation of China (Grant Nos. 31228022 and 31372430), the Special Fund for Agro-scientific Research in the Public Interest (Grant No. 201303042), the Open Funds of State Key Laboratory of Veterinary Etiological Biology, Lanzhou Veterinary Research Institute, Chinese Academy of Agricultural Sciences (SKLVEB2013KFKT006), and the Science Fund for Creative Research Groups of Gansu Province (Grant No. 1210RJIA006).

\section{Author details}

${ }^{1}$ State Key Laboratory of Veterinary Etiological Biology, Key Laboratory of Veterinary Parasitology of Gansu Province, Lanzhou Veterinary Research Institute, Chinese Academy of Agricultural Sciences, Lanzhou, Gansu Province 730046, PR China. ${ }^{2}$ Military Veterinary Institute, Academy of Military Medical Sciences, Key Laboratory of Jilin Province for Zoonosis Prevention and Control, Changchun, Jilin Province 130122, PR China. ${ }^{3}$ College of Animal Science and Technology, Jilin Agricultural University, Changchun, Jilin Province 130118, PR China. ${ }^{4}$ Jiangsu Co-innovation Center for the Prevention and Control of Important Animal Infectious Diseases and Zoonoses, Yangzhou University College of Veterinary Medicine, Yangzhou, Jiangsu Province 225009, PR China.

\section{Received: 28 August 2014 Accepted: 23 November 2014}

Published online: 03 December 2014

\section{References}

1. Dubey JP: Toxoplasmosis of Animals and Humans. 2nd edition. Boca Raton, Florida: CRC Press; 2010:313.

2. Tian YM, Dai FY, Huang SY, Deng ZH, Duan G, Zhou DH, Yang JF, Weng YB, Zhu XQ, Zou FC: First report of Toxoplasma gondii seroprevalence in peafowls in Yunnan Province, Southwestern China. Parasit Vectors 2012, 5:205.

3. Zhou P, Chen Z, Li HL, Zheng H, He S, Lin RQ, Zhu XQ: Toxoplasma gondii infection in humans in China. Parasit Vectors 2011, 4:105

4. Montoya JGLO: Toxoplasmosis. Lancet 2004, 363:1965-1976.

5. Dubey J, Jones J: Toxoplasma gondii infection in humans and animals in the United States. Int J Parasitol 2008, 38:1257-1278.

6. Yu L, Shen J, Su C, Sundermann CA: Genetic characterization of Toxoplasma gondii in wildlife from Alabama, USA. Parasitol Res 2013, 112:1333-1336.

7. Smith I, Wang LF: Bats and their virome:an important source of emerging viruses capable of infecting humans. Curr Opin Virol 2013, 3:84-91.

8. Calisher $\mathrm{CH}$, Childs JE, Field HE, Holmes KV, Schountz T: Bats: important reservoir hosts of emerging viruses. Clin Microbiol Rev 2006, 19:531-545.

9. De Lima H, Rodriguez N, Barrios MA, Avila A, Canizales I, Gutierrez S: Isolation and molecular identification of Leishmania chagasi from a bat (Carollia perspicillata) in northeastern Venezuela. Mem Inst Oswaldo Cruz 2008, 103:412-414.

10. Mühldorfer K: Bats and bacterial pathogens: a review. Zoonoses Public Health 2013, 60:93-103.

11. Wang W, Cao L, He B, Li J, Hu T, Zhang F, Fan Q, Tu C, Liu Q: Molecular characterization of Cryptosporidium in bats from Yunnan province, southwestern China. J Parasitol 2013, 99:1148-1150.

12. Dodd NS, Lord JS, Jehle R, Parker S, Parker F, Brooks DR, Hide G: Toxoplasma gondii: prevalence in species and genotypes of British bats (Pipistrellus pipistrellus and P. pygmaeus). Exp Parasitol 2014, 139:6-11.

13. Sun H, Wang Y, Zhang Y, Ge W, Zhang F, He B, Li Z, Fan Q, Wang W, Tu C, Li J, Liu Q: Prevalence and genetic characterization of Toxoplasma gondii in bats in Myanmar. Appl Environ Microbiol 2013, 79:3526-3528.

14. Sangster C, Gordon A, Hayes D: Systemic toxoplasmosis in captive flying-foxes. Aust Vet J 2012, 90:140-142.
15. Cabral AD, Gama AR, Sodre MM, Savani ES, Galvao-Dias MA, Jordao LR, Maeda MM, Yai LE, Gennari SM, Pena HF: First isolation and genotyping of Toxoplasma gondii from bats (Mammalia: Chiroptera). Vet Parasitol 2013, 193:100-104.

16. Schmidt S, Galvao A, Fernandes W, Oliveira R: Do primeiro encontro do Toxoplasma gondii (Nicolle \& Manceaux, 1909) em morcegos. Rev Goiana Med 1969, 15:149-154.

17. Yuan ZG, Luo SJ, Dubey JP, Zhou DH, Zhu YP, He Y, He XH, Zhang XX, Zhu $X Q$ : Serological evidence of Toxoplasma gondii infection in five species of bats in China. Vector Borne Zoonotic Dis 2013, 13:422-424.

18. Jiang HH, Qin SY, Wang W, He B, Hu TS, Wu JM, Fan QS, Tu CC, Liu Q, Zhu XQ: Prevalence and genetic characterization of Toxoplasma gondii infection in bats in southern China. Vet Parasitol 2014, 203:318-321.

19. Zhang XX, Lou ZZ, Huang SY, Zhou DH, Jia WZ, Su C, Zhu XQ: Genetic characterization of Toxoplasma gondii from Qinghai vole, Plateau pika and Tibetan ground-tit on the Qinghai-Tibet Plateau, China. Parasit Vectors 2013, 6:291.

20. Jiang HH, Huang SY, Zhou DH, Zhang XX, Su C, Deng SZ, Zhu XQ: Genetic characterization of Toxoplasma gondii from pigs from different localities in China by PCR-RFLP. Parasit Vectors 2013, 6:227.

21. Cong W, Meng QF, Song HQ, Zhou DH, Huang SY, Qian AD, Su C, Zhu XQ: Seroprevalence and genetic characterization of Toxoplasma gondii in three species of pet birds in China. Parasit Vectors 2014, 7:152.

22. Zhang XX, Huang SY, Zhang YG, Zhang Y, Zhu XQ, Liu Q: First report of genotyping of Toxoplasma gondii in free-living Microtus fortis in Northeastern China. J Parasitol 2014, 100:692-694.

23. Zhou P, Sun XT, Yin CC, Yang JF, Yuan ZG, Yan HK, Zhu XQ, Zou FC: Genetic characterization of Toxoplasma gondii isolates from pigs in southwestern China. J Parasitol 2011, 97:1193-1195.

24. Qian W, Wang H, Su C, Shan D, Cui X, Yang N, Lv C, Liu Q: Isolation and characterization of Toxoplasma gondii strains from stray cats revealed a single genotype in Beijing, China. Vet Parasitol 2012, 187:408-413.

25. Tian YM, Huang SY, Miao Q, Jiang HH, Yang JF, Su C, Zhu XQ, Zou FC: Genetic characterization of Toxoplasma gondii from cats in Yunnan Province, Southwestern China. Parasit Vectors 2014, 7:178.

26. Dubey JP, Zhu XQ, Sundar N, Zhang H, Kwok OC, Su C: Genetic and biologic characterization of Toxoplasma gondii isolates of cats from China. Vet Parasitol 2007, 145:352-356.

27. Zhou P, Zhang $H$, Lin $R Q$, Zhang DL, Song HQ, Su C, Zhu XQ: Genetic characterization of Toxoplasma gondii isolates from China. Parasitol Int 2009, 58:193-195.

28. Wang L, Chen H, Liu D, Huo X, Gao J, Song X, Xu X, Huang K, Liu W, Wang Y, Lu F, Lun ZR, Luo Q, Wang X, Shen J: Genotypes and mouse virulence of Toxoplasma gondii isolates from animals and humans in China. PLOS One 2013, 8:e53483.

29. Shwab EK, Zhu XQ, Majumdar D, Pena HF, Gennari SM, Dubey JP, Su C: Geographical patterns of Toxoplasma gondii genetic diversity revealed by multilocus PCR-RFLP genotyping. Parasitology 2014, 141:453-461.

30. Huang SY, Cong W, Zhou P, Zhou DH, Wu SM, Xu MJ, Zou FC, Song HQ, Zhu XQ: First report of genotyping of Toxoplasma gondii isolates from wild birds in China. J Parasitol 2012, 98:681-682.

31. Zhou P, Nie H, Zhang LX, Wang HY, Yin CC, Su C, Zhu XQ, Zhao JL: Genetic characterization of Toxoplasma gondii isolates from pigs in China. J Parasitol 2010, 96:1027-1029.

\section{doi:10.1186/s13071-014-0558-7}

Cite this article as: Qin et al:: Molecular detection and genotypic characterization of Toxoplasma gondii infection in bats in four provinces of China. Parasites \& Vectors 2014 7:558. 